\title{
XXIV. On the cooling of air by radiation and conduction, and on the propagation of sound
}

\section{Lord Rayleigh F.R.S.}

To cite this article: Lord Rayleigh F.R.S. (1899) XXIV. On the cooling of air by radiation and conduction, and on the propagation of sound , Philosophical Magazine Series 5, 47:286, 308-314, DOI: $10.1080 / 14786449908621263$

To link to this article: http://dx.doi.org/10.1080/14786449908621263

曲 Published online: 08 May 2009.

Submit your article to this journal $[\pi$

Џ Article views: 7

Q View related articles $\square$ 
light transmitted through half a centimetre or a few centimetres of sodium-vapour, of the same density as the densest part of the sodium-vapour in the portion of the solar atmosphere traversed by the light analysed in any particular observation. The question of temperature cannot occur except in so far as the density of the vapour, and the clustering in groups of atoms, or non-clustering (mist or vapour of sodium), are concerned.

\$ 8. A grand inference from the experimental foundation of Stokes' and Kirchhoff's original idea is that the periods of molecular vibration are the same to an exceedingly minute degree of accuracy through the great differences of range of vibration presented in the radiant molecules of an electric spark, electric arc, or flame, and in the molecules of a comparatively cool vapour or gas giving dark lines in the spectrum of light transmitted through it.

$\S 9$. It is much to be desired that laboratory experiments be made, notwithstanding their extreme difficulty, to determine the density and pressure of sodium-vapour through a wide range of temperature, and the relation between density, pressure, and temperature of gaseous sodium.

XXIV. On the Cooling of Air by Radiation and Conduction, and on the Propagation of Sound. By Lord RAYLEIGH, F.R.S.*

A CCORDING to Laplace's theory of the propagation of A Sound the expansions (and contractions) of the air are supposed to take place without transfer of heat. Many years ago $\mathrm{Sir} G$. Stokes † discussed the question of the influence of radiation from the heated air upon the propagation of sound. He showed that such small radiating power as is admissible would tell rather upon the intensity than upon the velocity. If $x$ be measured in the direction of propagation, the factor expressing the diminution of amplitude is $e^{-m x}$, where

$$
m=\frac{\gamma-1}{\gamma} \frac{q}{2 a} . \text {. . . . . . }
$$

In (1) $\gamma$ represents the ratio of specific heats $(1 \cdot 41), a$ is the velocity of sound, and $q$ is such that $e^{-q t}$ represents the law of cooling by radiation of a small mass of air maintained at constant volume. If $\tau$ denote the time required to traverse the distance $x, \tau=x / a$, and (1) may be taken to assert that the amplitude falls to any fraction, e.g. one-halt, of its original

* Communicated by the Author.

+ Phil. Mag. [4] i. p. 305, 1851; Theory of Sound, $\$ 247$. 
and Conduction, and on the Propagation of Sound. $\quad 309$

value in 7 times the interval of time required by a mass of air to cool to the same fraction of its original excess of temperature. "There appear to be no data by which the latter interval can be fixed with any approach to precision; but if we take it at one minute, the conclusion is that sound would be propagated for (seven) minutes, or travel over about (80) miles, without rery serious loss from this cause "*. We shall presently return to the consideration of the probable value of $q$.

Besides radiation there is also to be considered the influence of conductivity in causing transfer of heat, and further there are the effects of viscosity. The problems thus suggested have been solved by Stokes and Kirchhoff $\dagger$. If the law of propagation be

then

$$
u=e^{-m^{\prime} x} \cos (n t-x / a), \quad . \quad . \quad . \quad . \quad .
$$

$$
m^{\prime}=\frac{n^{2}}{2 a^{3}}\left\{\frac{4}{3} \mu^{\prime}+\nu \frac{\gamma-1}{\gamma}\right\}, . . . . .
$$

in which the firequency of vibration is $n / 2 \pi, \mu^{\prime}$ is the kinematic viscosity, and $\nu$ the thermometric conductivity. In C.G.S. measure we may take $\mu^{\prime}=\cdot 14, \nu=\cdot 26$, so that

$$
\frac{4}{3} \mu^{\prime}+v \frac{\gamma-1}{\gamma}=\cdot 25 \text {. }
$$

To take a particular case, let the frequency be 256 ; then since $a=33200$, we find for the time of propagation during which the amplitude diminishes in the ratio of $e: 1$,

$$
\left(m^{\prime} a\right)^{-1}=3560 \text { seconds. }
$$

Accordingly it is only very high sounds whose propagation can be appreciably influenced by viscosity and conductivity.

If we combine the effects of radiation with those of viscosity and conduction, we have as the factor of attenuation

$$
e^{-\left(m+m^{\prime}\right) x}
$$

where

$$
m+m^{\prime}=\cdot 14(q / a)+\cdot 12\left(n^{2} / a^{3}\right)
$$

In actual observations of sound we must expect the intensity to fall off in accordance with the law of inverse squares of distances. A very little experience of moderately distant sounds shows that in fact the intensity is in a high degree uncertain. These discrepancies are attributable to

- Proc. Roy. Inst., April 9, 1879.

† Pogg. Ann. vol. cxxxiv. p. 177, 1868; Theory of Sound, 2nd ed., $\$ 348$. 


\section{Lord Rayleigh on the Cooling of Air by Radiation}

atmospheric refraction and reflexion, and they are sometimes very surprising. But the question remains whether in a uniform condition of the atmosphere the attenuation is sensibly more rapid than can be accounted for by the law of inverse squares. Some interesting experiments towards the elucidation of this matter have been published by $\mathrm{Mr}$. Wilmer Duff *, who compared the distances of audibility of sounds proceeding respectively from two and from eight similar whistles. On an average the eight whistles were audible only about one-fourth further than a pair of whistles ; whereas, if the sphericity of the waves had been the only cause of attenuation, the distances would have been as 2 to 1 . Mr. Duff considers that in the circumstances of his experiments there was little opportunity for atmospheric irregularities, and he attributes the greater part of the falling off to radiation. Calculating from (1) he deduces a radiating power such that a mass of air at any given excess of temperature above its surroundings will (if its volume remain constant) fall by radiation to one-half of that excess in about one-twelfth of a second.

In this paper I propose to discuss further the question of the radiating power of air, and $I$ shall contend that on various grounds it is necessary to restrict it to a value hundreds of iimes smaller than that above mentioned. On this view Mr. Duff's results remain unexplained. For myself I should still be disposed to attribute them to atmospheric refraction. If further experiment should establish a rate of attenuation of the order in question as applicable in uniform air, it will I think be necessary to look for a cause not hitherto taken into account. We might imagine a delay in the equalization of the different sorts of energy in a gas undergoing compression, not wholly insensible in comparison with the time of vibration of the sound. If in the dynamical theory we assimilate the molecules of a gas to bard smooth bodies which are nearly but not absolutely spherical, and trace the effect of a rapid compression, we see that at the first moment the increment. of energy is wholly translational and thus produces a maximum effect in opposing the compression. A little later a due proportion of the excess of energy will have passed into rotational forms which do not influence the pressure, and this will accordingly fall off. Any effect of the kind must give rise to dissipation, and the amount of it will increase with the time required for the transformations, i.e. in the above mentioned illustration with the degree of approximation to the spherical form. In the case of absolute spheres no transformation of translatory into rotatory energy, or vice versa, would

* Phys. Review, vol. vi. p. 129, 1898. 
and Conduction, and on the Propagation of Sound. 311

occur in a finite time. There appears to be nothing in the behaviour of gases, as revealed to us by experiment, which forbids the supposition of a delay capable of influencing the propagation of sound.

Returning now to the question of the radiating power of air, we may establish a sort of superior limit by an argument based upon the theory of exchanges, itself firmly established by the researches of B. Stewart. Consider a spherical mass of radius $r$, slightly and uniformly heated. Whatever may be the radiation proceeding from a unit of surface, it must be less than the radiation from an ideal black surface under the same conditions. Let us, however, suppose that the radiation is the same in both cases and inquire what would then be the rate of cooling. According to Bottomley* the emissivity of a blackened surface moderately heated is 0001 . This is the amount of heat reckoned in water-gram-degree units emitted in one second from a square centimetre of surface heated $1^{\circ} \mathrm{C}$. If the excess of temperature be $\theta$, the whole emission is

$$
\theta \times 4 \pi r^{2} \times 0001 \text {. }
$$

On the other hand, the capacity for heat is

$$
\frac{4}{3} \pi r^{3} \times \cdot 0013 \times \cdot 24 \text {, }
$$

the first factor being the volume, the second the density, and the third the specific heat of air referred as usual to water. Thus for the rate of cooling,

whence

$$
\begin{array}{r}
\frac{d \theta}{\theta d t}=-\frac{\cdot 0003}{\cdot 0013 \times 24 \times r}=-\frac{1}{r} \text { very nearly, } \\
\theta=\theta_{0} e^{-t / r}, . . . . . . .
\end{array}
$$

$\theta_{0}$ being the initial value of $\theta$. The time in seconds of cooling in the ratio of $e: 1$ is thus represented numerically by $r$ expressed in centims.

When $r$ is very great, the suppositions on which (5) is calculated will be approximately correct, and that equation will then represent the actual law of cooling of the sphere of air, supposed to be maintained uniform by mixing if necessary. But ordinary experience, and more especially the observations of Tyndall upon the diathermancy of air, would lead us to suppose that this condition of things would not be approached until $r$ reached 1000 or perhaps 10,000 centims. For values of $r$ comparable with the half wave-length of ordinary sounds, e.g. 30 centim., it would seem that the real time of cooling must be a large multiple of that given by (5).

* Everett, C.G.S. Units, 1891, p. 134. 


\section{Lord Rayleigh on the Cooling of Air by Radiation}

At this rate the time of cooling of a mass of air must exceed, and probably largely exceed, 60 seconds. To suppose that this time is one-twelfth of a second would require a sphere of air 2 millim. in diameter to radiate as much heat as if it were of blackened copper at the same temperature.

Although, if the above argument is correct, there seems little likelihood of the cooling of moderate masses of air being sensibly influenced by radiation, I thought it would be of interest to inquire whether the observed cooling (or heating) in an experiment on the lines of Clement and Desormes could be adequately explained by the conduction of heat from the walls of the vessel in accordance with the known conductivity of air. A nearly spherical vessel of glass of about 35 centim. diameter, well encased, was fitted, air-tight, with two tubes. One of these led to a manometer charged with water or sulphuric acid; the other was provided with a stopcock and connected with an air-pump. In making an experiment the stopcock was closed and a vacuum established in a limited volume upon the further side. A rapid opening and reclosing of the cock allowed a certain quantity of air to escape suddenly, and thus gave rise to a nearly uniform cooling of that remaining behind in the vessel. At the same moment the liquid rose in the manometer, and the observation consisted in noting the times (given by a metronome beating seconds) at which the liquid in its descent passed the divisions of a scale, as the air recovered the temperature of the containing vessel. The first record would usually be at the third or fourth second from the turning of the cock, and the last after perhaps 120 seconds. In this way data are obtained for a plot of the curve of pressure; and the part actually observed has to be supplemented by extrapolation, so as to go back to the zero of time (the moment of turning the tap) and to allow for the drop which might occur subsequent to the last observation. An estimate, which cannot be much in error, is thus obtained of the whole rise in pressure during the recovery of temperature, and for the time, reckoned from the commencement, at which the rise is equal to one-half of the total.

In some of the earlier experiments the whole rise of pressure (fall in the manometer) during the recovery of temperature was about 20 millim. of water, and the time of half recovery was 15 seconds. I was desirous of working with the minimum range, since only in this way could it be hoped to eliminate the effect of gravity, whereby the interior and still cool parts of the included air would be made to fall and so come into closer proximity to the walls, and thus 
accelerate the mean cooling. In order to diminish the disturbance due to capillarity, the bore of the manometer-tube, which stood in a large open cistern, was increased to about 18 millim.*, and suitable optical arrangements were introduced to render small movements easily visible. By degrees the range was diminished, with a prolongation of the time of half recovery to $18,22,24$, and finally to about 26 seconds. The minimum range attained was represented by 3 or 4 millim. of water, and at this stage there did not appear to be much further prolongation of cooling in progress. There seemed to be no appreciable difference whether the air was artificially dried or not, but in no case was the moisture sufficient to develop fog under the very small expansions employed. The result of the experiments may be taken to be that when the influence of gravity was, as far as practicable, eliminated, the time of half recovery of temperature was about 26 seconds.

It may perhaps be well to give an example of an actual experiment. Thus in one trial on Nov. 1, the recorded times of passage across the divisions of the scale were $3,6,11,18$, $26,35,47,67,114$ seconds. The divisions themselves were millimetres, but the actual movements of the meniscus were less in the proportion of about $2 \frac{1}{2}: 1$. A plot of these numbers shows that one division must be added to represent the movement between $0^{\mathrm{s}}$ and $3^{\mathrm{s}}$, and about as much for the movement to be expected between $114^{\mathrm{s}}$ and $\infty$. The whole range is thus 10 divisions (corresponding to 4 millim. at the meniscus), and the mid point occurs at $26^{\mathrm{s}}$. On each occasion 3 or 4 sets of readings were taken under given conditions with fairly accordant results.

It now remains to compare with the time of heating derived from theory. The calculation is complicated by the consideration that when during the process any part becomes heated, it expands and compresses all the other parts, thereby developing heat in them. From the investigation which follows $\uparrow$, we see that the time of half recovery $t$ is given by the formula

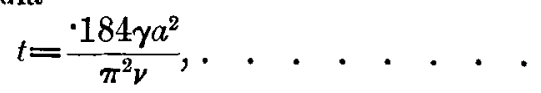

in which $a$ is the radius of the sphere, $\gamma$ the ratio of specific heats $(1 \cdot 41)$, and $v$ is the thermometric conductivity, found by dividing the ordinary or calorimetric conductivity by the

* It must not be forgotten that too large a diameter is objectionable, as leading to an augmentation of volume during an experiment, as the liquid falls.

+ See next paper. 


\section{Iord Rayleigh on Conduction of Heat in a Spherical}

thermal capacity of unit volume. This thermal capacity is to be taken with volume constant, and it will be less than the thermal capacity with pressure constant in the ratio of $y: 1$. Accordingly $v / \gamma$ in (6) represents the latter thermal capacity, of which the experimental value is $\cdot 00128 \times \cdot 239$, the first factor representing the density of air referred to water. Thus, if we take the calorimetric conductivity at $\cdot 000056$. we have in c.G.s. measure

and thence

$$
\nu=\cdot 258, \quad \nu / \gamma=\cdot 183 ;
$$

$$
t=\cdot 102 a^{2} \text {. }
$$

In the present apparatus $a$, determined by the contents, is 16.4 centim., whence

$$
t=27 \cdot 4 \text { seconds. }
$$

The agreement of the observed and calculated values is quite as close as could have been expected, and confirms the view that the transfer of heat is due to conduction, and that the part played by radiation is insensible. From a comparison of the experimental and calculated curves, however, it seems probable that the effect of gravity was not wholly eliminated, and that the later stages of the phenomenon, at any rate, may still have been a little influenced by a downward movement of the central parts.

XXV. On the Conduction of Heat in a Spherical Mass of Air confined by Walls at a Constant Temperature. By Lord RAYLEIGH, $\boldsymbol{F} . R . S . *$

$\mathrm{T} \mathrm{T}$ is proposed to investigate the subsidence to thermal 1 equilibriam of a gas slightly disturbed therefrom and included in a solid vessel whose walls retain a constant temperature. The problem differs from those considered by Fourier in consequence of the mobility of the gas, which may give rise to two kinds of complication. In the first place gravity; taking advantage of the different densities prevailing in various parts, tends to produce circulation. In many cases the subsidence to equilibrium must be greatly modified thereby. But this effect diminishes with the amount of the temperature disturbance, and for infinitesimal disturbances the influence of gravity disappears. On the other hand, the second complication remains, even though we limit ourselves to infinitesimal disturbances. When one part of the gas expands in consequence of reception of heat by

* Communicated by the Aulhor. 